International Journal of Pure and Applied Mathematics

Volume 89 No. 4 2013, 483-496

ISSN: 1311-8080 (printed version); ISSN: 1314-3395 (on-line version)

url: http://www.ijpam.eu

doi: http://dx.doi.org/10.12732/ijpam.v89i4.4

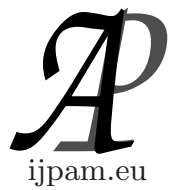

\title{
ON $I$-CAUCHY SEQUENCES IN $p$-ADIC LINEAR 2-NORMED SPACES
}

\author{
B. Surender Reddy ${ }^{1}$, D. Shankaraiah ${ }^{2}$ \\ ${ }^{1}$ Department of Mathematics \\ University College of Science \\ Saifabad, Osmania University \\ Hyderabad, AP, INDIA \\ ${ }^{2}$ Department of Mathematics \\ CVR College of Engineering \\ Ibrahimpatnam, Ranga Reddy District, AP, INDIA
}

\begin{abstract}
In this paper, we introduce the concept of $I^{*}$-convergence which is closely related to $I$-convergence and the concepts $I$ and $I^{*}$-Cauchy sequences in $p$-adic linear 2-normed space. Also we investigate the relation between these concepts in $p$-adic linear 2-normed space.
\end{abstract}

AMS Subject Classification: 11B68, 11F80, 46A15, 11S80, 46A65

Key Words: 2-normed space, $p$-adic 2-norm, $p$-adic linear 2-normed space, $I$-Cauchy sequence, $I^{*}$-Cauchy sequence

\section{Introduction}

In 1850, Kummer introduced the $p$-adic numbers. Then the German Mathematician, Kurt Hensel (1861-1941) developed the $p$-adic numbers which was concerned with the development of algebraic numbers in power series, around the end of the nineteenth century in 1897 . Then $p$-adic numbers were generalized to ordinals (or valuation) by Kürschak in 1913, and Minkowski (1884), Tate (1960), Kubota-Leopoldt (1964), Iwasawa, Serre, Mazur, Manin, Katz,

Received: August 8, 2013

(C) 2013 Academic Publications, Ltd.

${ }^{\S}$ Correspondence author url: www.acadpubl.eu 
and the others. There are numbers of all kinds such as rational, real, complex, $p$-adic numbers. Hensel's p-adic's numbers are now widely used in many fields such as analysis, physics and computer science. The $p$-adic numbers are less well known than the others, but they play a fundamental role in number theory in other parts of mathematics. Although, they have penatrated several mathematical fields, among them, number theory, algebraic geometry, algebraic topology and analysis. These numbers are now well-established in mathematical world and used more and more by physicists as well. Over the last century $p$-adic numbers and $p$-adic analysis have come to play an important role in number theory. They have many applications in mathematics, for example: Representation theory, algebraic geometry, and modern number theory and many applications in mathematical physics since 1897, for example; String theory, QFT, quantum mechanics, dynamical systems, complex systems, etc. Recently, Branko Dragovich in his study ([4]) he constructed $p$-adic approach to the genetic code and the genome and gave a new approach between $p$-adic fields and biology with chemistry, especially organic chemistry. The other researchers gave the different approach with $p$-adic on various disciplines of mathematics and its allied subjects ( see ([1], [2], [3], [10], [11], [12], [13], [16], [23], [27], [28]) for more details).

The concept of linear 2-normed spaces has been investigated by Gähler in 1965 ([5]) and has been developed extensively in different subjects by others. Lewandowska published a series of papers on 2-normed sets and generalized 2-normed spaces, convergent sequences, 2-Banach spaces,...etc. (see ([9], [17], [18], [19], [22], for more details). The notion of ideal convergence was introduced first by P.Kostyrko et al [15] as an interesting generalization of statistical convergence. The concept of an $I$-cluster point and $I$-limit point of a sequence in metric space was introduced and some results for the set of $I$-cluster points and $I$-limit points obtained in [14]. A.Sahiner et al [24] introduced $I$-cluster points convergence sequences in 2-normed linear spaces and and Gurdal [7] investigated the relation between $I$-cluster points and ordinary limit points of sequences in 2-normed spaces. The concept of $I$-Cauchy sequences in 2-normed spaces was introduced by Gurdal and Isil Acik [8], [21].

Mehmet Acikgoz ([20]) introduced a very understandable and readable connection between the concepts in $p$-adic numbers, $p$-adic analysis and linear 2normed spaces. B.Surender Reddy [25] introduced some properties of $p$-adic linear 2-normed spaces and obtain necessary and sufficient conditions for $p$-adic 2-norms to be equivalent on $p$-adic linear 2-normed spaces. Recently B.Surender Reddy and D.Shankaraiah [26] introduced ideal convergent sequences in $p$-adic linear 2-normed spaces. 
The main aim of this paper is to introduce the concept of $I^{*}$-convergence which is closely related to $I$-convergence and the concepts $I$ and $I^{*}$-Cauchy sequences in $p$-adic linear 2-normed space $\left(X, N(\bullet, \bullet)_{p}\right)$. Also we investigate the relation between these concepts in $p$-adic linear 2-normed spaces.

\section{Preliminaries}

In this paper, we will use the notations, $p$ for a prime number, $Z$ - the ring of rational integers, $Z^{+}$- the positive integers, $Q$ - the field of rational numbers, $R$ - the field of real numbers, $R^{+}$- the positive real numbers, $Z_{p}$ - the ring of $p$-adic rational integers, $Q_{p}$ - the field of $p$-adic rational numbers, $C$ - the field of complex numbers and $C_{p}$ - the p-adic completion of the algebraic closure of $Q_{p}$.

Definition 1. (i) The $p$-adic ordinal (valuation) of $x$ and $y$, for $0 \neq x, y \in$ $Z$ is $\operatorname{ord}_{p}(x, y)=\max \left\{r: p^{r} / x\right.$ and $\left.p^{r} / y\right\} \geq 0$

(ii) For $\frac{a}{b}, \frac{c}{d} \in Q$, the $p$-adic value of $\frac{a}{b}$ and $\frac{c}{d}$ is

$\operatorname{ord}_{p}\left(\frac{a}{b}, \frac{c}{d}\right)=\operatorname{ord}_{p}(a, c)-\operatorname{ord}_{p}(a, d)-\operatorname{ord}_{p}(b, c)+\operatorname{ord}_{p}(b, d)$

(iii) For $\frac{a}{b}, c \in Q$, with $d=1$, the $p$-adic value of $\frac{a}{b}$ and $c$ is $\operatorname{ord}_{p}\left(\frac{a}{b}, c\right)=$ $\operatorname{ord}_{p}(a, c)-\operatorname{ord}_{p}(b, c)$

Notice that in all cases, ord $_{p}$ in 2-norm, gives an integer and that for rational number $\frac{a}{b}$ and $\frac{c}{d}$ the value of $\operatorname{ord}_{p}\left(\frac{a}{b}, \frac{c}{d}\right)$ is well defined. i.e., if $\frac{a}{b}=\frac{a^{\prime}}{b^{\prime}}$ and $\frac{c}{d}=\frac{c^{\prime}}{d^{\prime}}$ then $\operatorname{ord}_{p}\left(\frac{a}{b}, \frac{c}{d}\right)=\operatorname{ord}_{p}\left(\frac{a^{\prime}}{b^{\prime}}, \frac{c^{\prime}}{d^{\prime}}\right)$. We also introduce the convention that $\operatorname{ord}_{p}(0, y)=\operatorname{ord}_{p}(x, 0)=\infty$. The $p$-adic valuation has the following properties

Proposition 2. For all $x, y \in Q$, we have for $\operatorname{ord}_{p}$;

(i) $\operatorname{ord}_{p}(x, y)=\infty \Longleftrightarrow x=0$ or $y=0$,

(ii) $\operatorname{ord}_{p}(x z, y)=\operatorname{ord}_{p}(x, y)+\operatorname{ord}_{p}(z, y)$,

(iii) $\operatorname{ord}_{p}(x+z, y) \geq \min \left\{\operatorname{ord}_{p}(x, y), \operatorname{ord}_{p}(z, y)\right\}$ and with equality when $\operatorname{ord}_{p}(x, y) \neq \operatorname{ord}_{p}(z, y)$.

Definition 3. Let $X$ be a linear space of dimension greater than 1 over $K$, where $K$ is the real or complex numbers field. Suppose $N(\bullet, \bullet)$ be a nonnegative real valued function on $X \times X$ satisfying the following conditions:

$(2 N 1): N(x, y)>0$ and $N(x, y)=0$ if and only if $x$ and $y$ are linearly dependent vectors,

$(2 N 2): N(x, y)=N(y, x)$ for all $x, y \in X$,

(2N3) : $N(\lambda x, y)=|\lambda| N(x, y)$ for all $\lambda \in K$ and $x, y \in X$,

$(2 N 4): N(x+y, z) \leq N(x, z)+N(y, z)$ for all $x, y, z \in X$. 
Then $N(\bullet, \bullet)$ is called a 2-norm on $X$ and the pair $(X, N(\bullet, \bullet))$ is called a linear 2-normed space.

Definition 4. For all $x, y \in Q$, let the $p$-adic norm of $x, y$ be given by $N(x, y)_{p}=p^{-o r d} d_{p}(x, y)$, if $x \neq 0$ and $y \neq 0$

$$
=p^{-\infty}=0 \text {, if } x=0 \text { or } y=0
$$

where $\operatorname{ord}_{p}(x, y)=\max \left\{r: p^{r} / x\right.$ and $\left.p^{r} / y\right\}$.

Proposition 5. Let the function $N(\bullet, \bullet)_{p}$ be a non-negative real valued function on $Q \times Q$ satisfying the following conditions:

$$
N(\bullet, \bullet)_{p}: Q \times Q \longrightarrow R^{+} \cup\{0\}=\{r: r \geq 0\}
$$

(i) $N(x, z)_{p}=0$ if and only if $x=0$ or $z=0$,

(ii) $N(x y, z)_{p}=N(x, z)_{p} N(y, z)_{p}$ for all $x, y$ and $z \in Q$,

(iii) $N(x+y, z)_{p} \leq \max \left\{N(x, z)_{p}, N(y, z)_{p}\right\}$ and with equality when $N(x, z)_{p} \neq$ $N(y, z)_{p}$, where $N(\bullet, \bullet)_{p}$ is a non-Archimedean norm on $Q$.

Let $N(x, z)_{p}$ be a non-negative real valued function defined on the rational numbers $Q \times Q$ such that $N(x, z)_{p}=0$ for $x=0$ or $z=0, N(x, z)_{p}>0$ when $x \neq 0, z \neq 0, N(x y, z)_{p}=N(x, z)_{p} N(y, z)_{p}$ for all $x, y, z \in Q$ and

$$
N(x+y, z)_{p} \leq K\left(N(x, z)_{p}+N(y, z)_{p}\right)
$$

for some $K \geq 1$ and all $x, y, z \in Q$. For the usual triangle inequality one ask that this condition holds with $K=1$, i.e.,

$$
N(x+y, z)_{p} \leq\left(N(x, z)_{p}+N(y, z)_{p}\right)
$$

for all $x, y, z \in Q$.

The ultrametric version of the triangle inequality is stronger still and asks that

$$
N(x+y, z)_{p} \leq \max \left(N(x, z)_{p}, N(y, z)_{p}\right)
$$

for all $x, y, z \in Q$. If $N(\bullet, \bullet)_{p}$ satisfies the equation (1), $n$ is a positive integer and $x_{1}, x_{2}, x_{3}, \ldots, x_{2^{n}} \neq 0, z \in Q$, then

$$
N\left(\sum_{k=1}^{2^{n}} x_{k}, z\right)_{p} \leq K^{n} \sum_{k=1}^{2^{n}} N\left(x_{k}, z\right)_{p}
$$

as one can check using induction on $n$. For all $a>0, N(x, z)_{p}^{a}$ is a non-negative real valued function on $Q \times Q$ which vanished at 0 , is positive at all nonzero 
$x \in Q$ and sends products to products. If $N(x, z)_{p}$ satisfies the equation (1), then

$$
N(x+y, z)_{p}^{a} \leq K^{a}\left(N(x, z)_{p}^{a}+N(y, z)_{p}^{a}\right)
$$

when $0<a \leq 1$ and

$$
N(x+y, z)_{p}^{a} \leq 2^{a-1} K^{a}\left(N(x, z)_{p}^{a}+N(y, z)_{p}^{a}\right)
$$

when $a \geq 1$.

In particular, if $N(x, z)_{p}$ satisfies the well-known triangle inequality (2) and $0<a \leq 1$, then $N(x, z)_{p}^{a}$ also satisfies the the well-known triangle inequality. If $N(x, z)_{p}$ satisfies the ultrametric version (3) of the triangle inequality, then $N(x, z)_{p}^{a}$ satisfies the ultrametric version of the triangle inequality for all $a \geq 0$.

Suppose a mapping $d_{p}: X \times X \times X \longrightarrow R$ on a non-empty set $X$ satisfying the following conditions, for all $x, y, z \in X$

$\left(D_{1}\right)$ For any two different elements $x$ and $y$ in $X$ there is an element $z$ in $X$ such that $d_{p}(x, y, z) \neq 0$

$\left(D_{2}\right) d_{p}(x, y, z)=0$ when two of three elements are equal

$\left(D_{3}\right) d_{p}(x, y, z)=d_{p}(x, z, y)=d_{p}(y, z, x)$

$\left(D_{4}\right) d_{p}(x, y, z) \leq d_{p}(x, y, w)+d_{p}(x, w, z)+d_{p}(w, y, z)$ for any $w$ in $X$.

Then $d_{p}$ is called $p$-adic 2-metric on $X$ and the pair $\left(X, d_{p}\right)$ is called $p$-adic 2-metric space.

If $p$-adic 2-metric also satisfies the condition

$d_{p}(x, y, z) \leq \max \left\{d_{p}(x, y, w), d_{p}(x, w, z), d_{p}(w, y, z)\right\}$ for $x, y, z, w \in X$, then $d_{p}$ is called a $p$-adic ultra 2 -metric and the pair $\left(X, d_{p}\right)$ is called a $p$-adic ultra 2-metric space.

Definition 6. Let $X$ be a linear space of dimension greater than 1 over $K$, where $K$ is the real or complex numbers field. Suppose $N(\bullet, \bullet)_{p}$ be a nonnegative real valued function on $X \times X$ satisfying the following conditions: $\left(2-p N_{1}\right): N(x, z)_{p}=0$ if and only if $x$ and $z$ are linearly dependent vectors, $\left(2-p N_{2}\right): N(x y, z)_{p}=N(x, z)_{p} N(y, z)_{p}$ for all $x, y, z \in X$, $\left(2-p N_{3}\right): N(x+y, z)_{p} \leq N(x, z)_{p}+N(y, z)_{p}$ for all $x, y, z \in X$, $\left(2-p N_{4}\right): N(\lambda x, z)_{p}=|\lambda| N(x, z)_{p}$ for all $\lambda \in K$ and $x, z \in X$.

Then $N(\bullet, \bullet)_{p}$ is called a $p$-adic 2-norm on $X$ and the pair $\left(X, N(\bullet, \bullet)_{p}\right)$ is called $p$-adic linear 2-normed space.

For every $p$-adic linear 2-normed space $\left(X, N(\bullet, \bullet)_{p}\right)$ the function defined on $X \times X \times X$ by $d_{p}(x, y, z)=N(x-z, y-z)_{p}$ is a $p$-adic 2-metric.Thus every $p$-adic linear 2-normed space $\left(X, N(\bullet, \bullet)_{p}\right)$ will be considered to be a $p$-adic 2-metric space with this 2-metric. A sequence $\left\{x_{n}\right\}_{n=1}^{\infty}$ of $p$-adic 2-metric space $\left(X, d_{p}\right)$ converges to $x \in X$ in $p$-adic 2-metric if for every $\epsilon>0$, there is an $\ell \geq 1$ 
such that $d_{p}\left(x_{n}, x, z\right)=N\left(x_{n}-z, x-z\right)_{p}<\epsilon$ for every $n \geq \ell$. For the given two sequences of $p$-adic 2-metric space $\left(X, d_{p}\right)$ which are $\left\{x_{n}\right\}_{n=1}^{\infty}$ and $\left\{y_{n}\right\}_{n=1}^{\infty}$ converges to $x, y \in X$ in the $p$-adic 2-metric respectively, then the sequence of sums $x_{n}+y_{n}$ and the product $x_{n} y_{n}$ converges to the sum $x+y$ and to the product $x y$ of the limits of initial sequences.

A sequence $\left\{x_{n}\right\}_{n=1}^{\infty}$ of $p$-adic 2-metric space $\left(X, d_{p}\right)$ is a Cauchy sequence with respect to the $p$-adic 2 -metric if for every $\epsilon>0$, there is an $\ell \geq 1$ such that $d_{p}\left(x_{n}, x_{m}, z\right)=N\left(x_{n}-z, x_{m}-z\right)_{p}<\epsilon$ for every $n, m \geq l$. A sequence $\left\{x_{n}\right\}_{n=1}^{\infty}$ in a $p$-adic linear 2-normed space $\left(X, N(\bullet, \bullet)_{p}\right)$ is convergent if there exists an $x \in X$ such that $\lim _{n \rightarrow \infty} N\left(x_{n}-x, z\right)_{p}=0$ for all $z \in X$. A sequence $\left\{x_{n}\right\}_{n \geq 1}$ in a $p$-adic linear 2-normed space $\left(X, N(\bullet, \bullet)_{p}\right)$ is a Cauchy sequence if for each $\varepsilon>0$, there is an $\ell \geq 1$ such that $N\left(x_{n}-x_{m}, z\right)_{p}<\varepsilon$, for all $n, m \geq \ell$ and for all $z \in X$. A $p$-adic linear 2-normed space $\left(X, N(\bullet, \bullet)_{p}\right)$ is complete if every Cauchy sequence is convergent in $p$-adic linear 2 -normed space. A $p$-adic linear 2-normed space $\left(X, N(\bullet, \bullet)_{p}\right)$ is a $p$-adic 2 -Banach space if $p$-adic linear 2-normed space is complete.

Proposition 7. If a sequence $\left\{x_{n}\right\}$ in a $p$-adic linear 2-normed space $\left(X, N(\bullet, \bullet)_{p}\right)$ is convergent to $x \in X$ then $\lim _{n \rightarrow \infty} N\left(x_{n}, z\right)_{p}=N(x, z)_{p}$ for each $z \in X$.

Proposition 8. If $\lim _{n \rightarrow \infty} N\left(x_{n}, z\right)_{p}$ exists then we say that $\left\{x_{n}\right\}_{n \geq 1}$ is a Cauchy sequence with respect to $N(\bullet, \bullet)_{p}$.

Proof. Let us suppose that $\lim _{n \rightarrow \infty} N\left(x_{n}, z\right)_{p}=x$. Then we can obtain a constant $M_{1}$ such that $n>M_{1} \Rightarrow N\left(x-x_{n}, z\right)_{p}<\frac{\varepsilon}{2}$. If $m, n>M_{1}$ then $N\left(x-x_{n}, z\right)_{p}<\frac{\varepsilon}{2}$ and $N\left(x-x_{m}, z\right)_{p}<\frac{\varepsilon}{2}$, hence by using the triangle inequality, we have $N\left(x_{m}-x_{n}, z\right)_{p}=N\left(x_{m}-x+x-x_{n}, z\right)_{p} \leq N\left(x_{m}-x, z\right)_{p}+$ $N\left(x-x_{n}, z\right)_{p}<\frac{\varepsilon}{2}+\frac{\varepsilon}{2}=\varepsilon$.

Definition 9. A sequence $\left\{x_{n}\right\}_{n \geq 1}$ is called a null sequence in $p$-adic linear 2-normed space if $\lim _{n \rightarrow \infty} N\left(x_{n}, z\right)_{p}=0$ for all $z \in X$.

Example 10. Let $x_{n}=p^{n}$ and $z=p^{r}$ with $r<n$ in the $p$-adic 2-norm over $X=Q$. Then

$$
\begin{aligned}
N\left(p^{n}, p^{r}\right)_{p} & =p^{-o r d}\left(p^{n}, p^{r}\right), \text { if } p^{n} \neq 0 \text { and } p^{r} \neq 0 \\
& =p^{-\infty}=0, \text { if } p^{n}=0 \text { or } p^{r}=0 .
\end{aligned}
$$

In this case $N\left(p^{n}, p^{r}\right)_{p}=p^{-n}=\frac{1}{p^{n}}=0$, as $n \rightarrow \infty$. Therefore, $\lim _{n \rightarrow \infty} N\left(x_{n}, z\right)_{p}$ $=0$, for all $z \in X$. Hence this sequence is a null sequence with respect to the $p$-adic 2-norm. 
Definition 11. A $p$-adic number $(\alpha, \beta)$ can be uniquely written in the form

$$
(\alpha, \beta)=\sum_{i=n, j=m}^{\infty}\left(a_{i} p^{i}, b_{j} p^{j}\right)
$$

where each $0 \leq a_{i}, b_{j} \leq p-1$ and $p$-adic 2 -norm of the number $(\alpha, \beta)$ is defined as $N(\alpha, \beta)_{p}=n,(n \in R)$ and the double series $\left(1+p+p^{2}+p^{3}+\ldots, 1+p+\right.$ $\left.p^{2}+p^{3}+\ldots\right)$ converges to $\frac{1}{1-p}$ in the $p$-adic 2 -norm.

\section{Main Results}

In this section, we introduce the concept of $I^{*}$-convergence which is closely related to $I$-convergence in $p$-adic linear 2-normed space $\left(X, N(\bullet, \bullet)_{p}\right)$ and we introduce the concepts $I$ and $I^{*}$-Cauchy sequences in $p$-adic linear 2-normed space $\left(X, N(\bullet, \bullet)_{p}\right)$. Also we investigate the relation between these concepts in $p$-adic linear 2-normed spaces.

A family of sets $I \subseteq 2^{Y}$ (power sets of $Y$ ) is said to be an ideal if $\Phi \in I, I$ is additive i.e., $A, B \in I \Longrightarrow A \cup B \in I$ and hereditary i.e., $A \in I, B \subseteq A \Longrightarrow$ $B \in I$.

A non empty family of sets $F \subset 2^{Y}$ is a filter on $Y$ if and only if $\Phi \notin F$, $A \cap B \in F$, for each $A, B \in F$, and any subset of an element of $F$ is in $F$. An ideal $I$ is called non-trivial if $I \neq \Phi$ and $Y \notin I$. Clearly $I$ is a non-trivial ideal if and only if $F=F(I)=\{Y-A: A \in I\}$ is a filter in $Y$, called the filter associated with the ideal $I$. A non-trivial ideal $I$ is called admissible if and only if $\{\{n\}: n \in Y\} \subset I$.

An admissible ideal $F \subset 2^{Y}$ is said to have the property (AP) if for any sequence $\left\{A_{1}, A_{2}, A_{3}, \ldots\right\}$ of mutually disjoint sets of $I$ there is a sequence $\left\{B_{1}, B_{2}, B_{3}, \ldots\right\}$ of sets such that each symmetric difference $A_{i} \triangle B_{i}, i=1,2,3, \ldots$ is finite and $B=\bigcup_{n=1}^{\infty} B_{i} \in I$.

Definition 12. Let $(X, N(\bullet, \bullet))$ be a linear 2-normed space. A sequence $\left\{x_{n}\right\}$ of elements of $X$ is called to be statistically convergent to $x \in X$ if the set $A(\varepsilon)=\left\{n \in N: N\left(x_{n}-x, z\right) \geq \varepsilon\right\}$ has natural density zero for each $\varepsilon>0$.

Definition 13. Let $I \subset 2^{N}$ be a non trivial ideal in $N$. The sequence $\left\{x_{n}\right\}$ of elements of linear 2-normed space $(X, N(\bullet, \bullet))$ is said to be $I$-convergent to $x \in X$ if for each $\varepsilon>0$ the set $A(\varepsilon)=\left\{n \in N: N\left(x_{n}-x, z\right) \geq \varepsilon\right\} \in I$.

Definition 14. Let $I \subset 2^{N}$ be a non trivial ideal in $N$. A sequence $\left\{x_{n}\right\}$ in a $p$-adic linear 2-normed space $\left(X, N(\bullet, \bullet)_{p}\right)$ is said to be $I$-convergent 
to $x \in X$ if for each $\varepsilon>0$ and non-zero $z$ in $X$ the set $A(\varepsilon)=\{n \in N$ : $\left.N\left(x_{n}-x, z\right)_{p} \geq \varepsilon\right\} \in I$. It is denoted by $I-\lim _{n \rightarrow \infty} N\left(x_{n}-x, z\right)_{p}=0$ or $I-\lim _{n \rightarrow \infty} N\left(x_{n}, z\right)_{p}=N(x, z)_{p}$ for each non-zero $z \in X$. The number $x$ is called $I$-limit of the sequence $\left\{x_{n}\right\}$.

Lemma 15. Let $\left\{P_{i}\right\}_{i=1}^{\infty}$ be a countable collection of subsets of $N$ such that $P_{i} \in F(I)$ for each $i$, where $F(I)$ is a filter associated by an admissible ideal $I$ with property (AP). Then there is a set $P \subset N$ such that $P \in F(I)$ and the set $P-P_{i}$ is finite for all $i$.

Lemma 16. Let $I \subset 2^{N}$ be an admissible ideal with property (AP) and $\left(X, N(\bullet, \bullet)_{p}\right)$ be a $p$-adic linear 2-normed space. If a sequence $\left\{x_{n}\right\}$ in $X$ is $I$-convergent to $x \in X$ then there exists a set $P \in F(I), P=\left\{m_{1}<m_{2}<\ldots<\right.$ $\left.m_{k} \cdots\right\}$ such that $\lim _{k \rightarrow \infty} N\left(x_{m_{k}}-x, z\right)_{p}=0$, for each non-zero $z \in X$.

Proof. Let $\left\{x_{n}\right\}$ be a sequence of elements in a $p$-adic linear 2-normed space $\left(X, N(\bullet, \bullet)_{p}\right)$ is $I$-convergent to $x \in X$. Then the set $A(\varepsilon)=\left\{n \in N: N\left(x_{n}-\right.\right.$ $\left.x, z)_{p} \geq \varepsilon\right\} \in I$ for each $\varepsilon>0$ and non- zero $z$ in $X$. Define $P_{i}=\{n \in N$ : $\left.N\left(x_{n}-x, z\right)_{p}<\frac{1}{i}\right\}$ and $H_{i}=N-P_{i}=\left\{n \in N: N\left(x_{n}-x, z\right)_{p} \geq \frac{1}{i}\right\}$ for each $i \in N$ and non-zero $z$ in $X$. Then $H_{i} \in I$ and $P_{i} \in F(I)$, for each $i \in N$. By applying Lemma (15), we get $P \in F(I)$ such that $P=\left\{m_{1}<m_{2}<\ldots<m_{k} \ldots\right\}$ and $P-P_{i}$ is finite set for all $i$. Now define the sequence $\left\{y_{n}\right\}$ in $X$ such that $y_{n}=x_{n}$ for each $n \in P$ and $y_{n}=x$ for $n \notin P$. Then $\lim _{n \rightarrow \infty} N\left(y_{n}-x, z\right)_{p}=0$ which implies that $\lim _{k \rightarrow \infty} N\left(x_{m_{k}}-x, z\right)_{p}=0$, for each non-zero $z \in X$.

Now we introduce the concept of $I^{*}$-convergence of sequences in $p$-adic linear 2 -normed spaces which is closely related to $I$-convergence of sequences in $p$-adic linear 2-normed spaces.

Definition 17. A sequence $\left\{x_{n}\right\}$ in a $p$-adic linear 2-normed space $(X, N(\bullet$, - $)_{p}$ ) is said to be $I^{*}$-convergent to $x \in X$ if and only if there exists a set $M \in F(I), M=\left\{m_{1}<m_{2}<\ldots<m_{k} \ldots\right\}$ such that $\lim _{k \rightarrow \infty} N\left(x_{m_{k}}-x, z\right)_{p}=0$, for each non-zero $z \in X$. It is denoted by $I^{*}-\lim _{n \rightarrow \infty} N\left(x_{n}-x, z\right)_{p}=0$ or $I^{*}-\lim _{n \rightarrow \infty} N\left(x_{n}, z\right)_{p}=N(x, z)_{p}$, for each non-zero $z$ in $X$. The number $x$ is called $I^{*}$-limit of the sequence $\left\{x_{n}\right\}$.

From Lemma (16) it is clear that if $I$ is an admissible ideal with property (AP) and $I-\lim _{n \rightarrow \infty} N\left(x_{n}-x, z\right)_{p}=0$ then $I^{*}-\lim _{n \rightarrow \infty} N\left(x_{n}-x, z\right)_{p}=0$, for each non-zero $z \in X$ i.e., if $I$ is an admissible ideal with property (AP) and a sequence 
$\left\{x_{n}\right\}$ is $I$-convergent to $x$ in a $p$-adic linear 2-normed space $\left(X, N(\bullet, \bullet)_{p}\right)$ then $\left\{x_{n}\right\}$ is $I^{*}$-convergent to $x$ in a $p$-adic linear 2-normed space $\left(X, N(\bullet, \bullet)_{p}\right)$.

Lemma 18. Let $I \subset 2^{N}$ be an admissible ideal. If $I^{*}-\lim _{n \rightarrow \infty} N\left(x_{n}-x, z\right)_{p}=$ 0 then $I-\lim _{n \rightarrow \infty} N\left(x_{n}-x, z\right)_{p}=0$ for each non-zero $z$ in a $p$-adic linear 2-normed space $\left(X, N(\bullet, \bullet)_{p}\right)$.

Proof. Let $I \subset 2^{N}$ be an admissible ideal and $I^{*}-\lim _{n \rightarrow \infty} N\left(x_{n}-x, z\right)_{p}=0$. Then there exists a set $H \in I$ such that for $M=N-H=\left\{m_{1}<m_{2}<\ldots<\right.$ $\left.m_{k} \ldots\right\}$ we have

$$
\lim _{k \rightarrow \infty} N\left(x_{m_{k}}-x, z\right)_{p}=0 \text {, for each non-zero } z \in X .
$$

Let $\varepsilon>0$, by virtue of equation (7) there exists $k_{0} \in N$ such that $N\left(x_{m_{k}}-\right.$ $x, z)_{p}<\varepsilon$ for each $k>k_{0}$. Then obviously

$$
A(\varepsilon)=\left\{n \in N: N\left(x_{n}-x, z\right)_{p} \geq \varepsilon\right\} \subset H \cup\left\{m_{1}<m_{2}<\ldots<m_{k_{0}}\right\}
$$

The set on the right hand side of (8) belongs to $I$ (since $I$ is an admissible). So $A(\varepsilon) \in I$. Therefore $I-\lim _{n \rightarrow \infty} N\left(x_{n}-x, z\right)_{p}=0$ for each non-zero $z \in X$.

From Lemma (16) and Lemma (18) we obtain the following Lemma which gives the equivalence between $I$-convergence and $I^{*}$-convergence in $p$-adic linear 2-normed space $\left(X, N(\bullet, \bullet)_{p}\right)$.

Lemma 19. Let $I \subset 2^{N}$ be an admissible ideal with property $(A P)$ and $\left(X, N(\bullet, \bullet)_{p}\right)$ be a $p$-adic linear 2-normed space. Then $I-\lim _{n \rightarrow \infty} N\left(x_{n}-x, z\right)_{p}=0$ for each non-zero $z \in X$ if and only if there exists a set $M \in F(I), M=\left\{m_{1}<\right.$ $\left.m_{2}<\ldots<m_{k} \ldots\right\}$ such that $\lim _{k \rightarrow \infty} N\left(x_{m_{k}}-x, z\right)_{p}=0$, for each non-zero $z \in X$. i.e., Let $I \subset 2^{N}$ is an admissible ideal with property $(A P)$ and $\left(X, N(\bullet, \bullet)_{p}\right)$ be a $p$-adic linear 2-normed space. Then $\left\{x_{n}\right\}$ is I-convergence to $x$ in $p$-adic linear 2-normed space $\left(X, N(\bullet, \bullet)_{p}\right)$ if and only if $\left\{x_{n}\right\}$ is $I^{*}$-convergent to $x$ in $p$-adic linear 2-normed space $\left(X, N(\bullet, \bullet)_{p}\right)$.

Now we introduce the concepts $I$ and $I$-Cauchy sequences in $p$-adic linear 2-normed space $\left(X, N(\bullet, \bullet)_{p}\right)$. Also, we investigate the relations between these concepts in $p$-adic linear 2-normed space $\left(X, N(\bullet, \bullet)_{p}\right)$.

Definition 20. Let $\left(X, N(\bullet, \bullet)_{p}\right)$ be a $p$-adic linear 2-normed space and $I \subset 2^{N}$ be an admissible ideal. The sequence $\left\{x_{n}\right\}$ in $X$ is said to be $I$-Cauchy sequence in $X$, if for each $\varepsilon>0$ and non-zero $z \in X$ there exists a number $\ell(\varepsilon, z)$ such that $\left\{k \in N: N\left(x_{k}-x_{\ell}, z\right)_{p} \geq \varepsilon\right\} \in I$. 
Definition 21. Let $\left(X, N(\bullet, \bullet)_{p}\right)$ be a $p$-adic linear 2-normed space and $I \subset 2^{N}$ be an admissible ideal. Then the sequence $\left\{x_{n}\right\}$ in $X$ is said to be $I^{*}$ Cauchy sequence in $X$ if there exists a set $M=\left\{m_{1}<m_{2}<\ldots<m_{k} \ldots\right\} \subset N$, $M \in F(I)$ such that the sequence $\left\{x_{m_{k}}\right\}$ is a Cauchy sequence in $X$. i.e., $\lim _{k, \ell \rightarrow \infty} N\left(x_{m_{k}}-x_{m_{\ell}}, z\right)_{p}=0$ for each non-zero $z \in X$.

Theorem 22. Let $I$ be an admissible ideal. If $\left\{x_{n}\right\}$ is $I^{*}$-Cauchy sequence then $\left\{x_{n}\right\}$ is $I$-Cauchy sequence in a $p$-adic linear 2-normed space $\left(X, N(\bullet, \bullet)_{p}\right)$.

Proof. Let $I$ be an admissible ideal and $\left\{x_{n}\right\}$ be $I^{*}$-Cauchy sequence in a $p$ adic linear 2-normed space $\left(X, N(\bullet, \bullet)_{p}\right)$. Then by definition there exists the set $M=\left\{m_{1}<m_{2}<\ldots<m_{k} \ldots\right\} \subset N, M \in F(I)$ such that $N\left(x_{m_{k}}-x_{m_{\ell}}, z\right)_{p}<\varepsilon$ for every non-zero $z \in X$ and $k, \ell>k_{0}(\varepsilon)$. Let $\ell(\varepsilon)=m_{k_{0}+1}$. Then for every $\varepsilon>0$, we have $N\left(x_{m_{k}}-x_{\ell}, z\right)_{p}<\varepsilon$, for every non-zero $z \in X$ and $k>k_{0}$. Now put $H=N-M$, it is clear that $H \in I$ and

$$
A(\varepsilon)=\left\{n \in N: N\left(x_{n}-x_{\ell}, z\right)_{p} \geq \varepsilon\right\} \subset H \cup\left\{m_{1}<m_{2}<\ldots<m_{k} \ldots\right\}
$$

Then the set on the R.H.S. of (9) belongs to $I$ because $I$ is an admissible ideal. Therefore for every $\varepsilon>0$ we find $\ell(\varepsilon)$ such that $A(\varepsilon) \in I$ i.e., $\left\{x_{n}\right\}$ is $I$-Cauchy sequence in a $p$-adic linear 2-normed space $\left(X, N(\bullet, \bullet)_{p}\right)$.

Now we will prove that $I^{*}$-convergence implies $I$-Cauchy condition in a $p$-adic linear 2-normed space $\left(X, N(\bullet, \bullet)_{p}\right)$.

Theorem 23. Let $I$ be an admissible ideal and $I^{*}-\lim _{n \rightarrow \infty} N\left(x_{n}-x, z\right)_{p}=0$ where $\left\{x_{n}\right\}$ is sequence in a $p$-adic linear 2-normed space $\left(X, N(\bullet, \bullet)_{p}\right)$ and $x \in$ $X$. Then $\left\{x_{n}\right\}$ is $I$-Cauchy sequence in $X$. In otherwords let $I$ be an admissible ideal and $\left(X, N(\bullet, \bullet)_{p}\right)$ be a $p$-adic linear 2-normed space. If a sequence $\left\{x_{n}\right\}$ in $X$ is $I^{*}$-convergent to $x \in X$, then $\left\{x_{n}\right\}$ is $I$-Cauchy sequence in $X$.

Proof. Let $I$ be an admissible ideal and $\left(X, N(\bullet, \bullet)_{p}\right)$ be a $p$-adic linear 2-normed space. Suppose a sequence $\left\{x_{n}\right\}$ in $X$ is $I^{*}$-convergent to $x \in X$. Then there exists the set $M \in F(I), M=\left\{m_{1}<m_{2}<\ldots<m_{k} \ldots\right\} \subset N$, such that $\lim _{k \rightarrow \infty} N\left(x_{m_{k}}-x, z\right)_{p}=0$, for each non-zero $z \in X$. It shows that there exists $k_{0}=k_{0}(\varepsilon)$ such that $N\left(x_{m_{k}}-x, z\right)_{p}<\frac{\varepsilon}{2}$ for every $\varepsilon>0$, nonzero $z \in X$ and $k>k_{0}$. N( $\left.x_{m_{k}}-x_{m_{\ell}}, z\right)_{p}=N\left(x_{m_{k}}-x+x-x_{m_{\ell}}, z\right)_{p}=$ $N\left(\left(x_{m_{k}}-x\right)-\left(x_{m_{\ell}}-x\right), z\right)_{p} \leq N\left(x_{m_{k}}-x, z\right)_{p}+N\left(x_{m_{l}}-x, z\right)_{p}<\frac{\varepsilon}{2}+\frac{\varepsilon}{2}=\varepsilon$, for every $\varepsilon>0$, non-zero $z \in X$ and $k>k_{0}, \ell>k_{0}$.

Therefore $\lim _{(k, \ell) \rightarrow \infty} N\left(x_{m_{k}}-x_{m_{\ell}}, z\right)_{p}=0 \Longrightarrow\left\{x_{m_{k}}\right\}$ is a Cauchy seqauence in $X$ 
$\Longrightarrow\left\{x_{n}\right\}$ is a $I^{*}$-Cauchy seqauence in $X$. By theorem (22), $\left\{x_{n}\right\}$ is a $I$-Cauchy seqauence in $X$

From Theorem (23) and Lemma (19) we have the following corollary which gives the relation between $I$-convergence and $I$-Cauchy sequence in a $p$-adic linear 2-normed space $\left(X, N(\bullet, \bullet)_{p}\right)$.

Corollary 24. Let $I \subset 2^{N}$ be an admissible ideal with property (AP). If $I-\lim _{n \rightarrow \infty} N\left(x_{n}-x, z\right)_{p}=0$, for each non- zero $z \in X$ then a sequence $\left\{x_{n}\right\}$ is $I$-Cauchy sequence in a $p$-adic linear 2-normed space $\left(X, N(\bullet, \bullet)_{p}\right)$ i.e, if $\left\{x_{n}\right\}$ is $I$-convergence to $x$ in a $p$-adic linear 2-normed space $\left(X, N(\bullet, \bullet)_{p}\right)$ then $\left\{x_{n}\right\}$ is $I$-Cauchy sequence in a $p$-adic linear 2-normed space $\left(X, N(\bullet, \bullet)_{p}\right)$.

Finally, we will give the following theorem which states that the equivalence of $I$-Cauchy sequence and $I^{*}$-Cauchy sequence in the case $I$ has the property (AP).

Theorem 25. Let $I$ be an admissible ideal with property (AP) and $\left(X, N(\bullet, \bullet)_{p}\right)$ be a $p$-adic linear 2-normed space. Then $\left\{x_{n}\right\}$ is $I$-Cauchy sequence in $X$ if and only if $\left\{x_{n}\right\}$ is $I^{*}$-Cauchy sequence in $X$.

Proof. By Theorem (22), if $\left\{x_{n}\right\}$ is $I^{*}$-Cauchy sequence then $\left\{x_{n}\right\}$ is $I$ Cauchy sequence in a $p$-adic linear 2 -normed space $\left(X, N(\bullet, \bullet)_{p}\right)$. Now we have to prove that $I$-Cauchy sequence is a $I^{*}$-Cauchy sequence in $X$. Let $\left\{x_{n}\right\}$ be Cauchy sequence in a $p$-adic linear 2-normed space $\left(X, N(\bullet, \bullet)_{p}\right)$. Then there exists $\ell(\varepsilon)$ such that $A(\varepsilon)=\left\{n \in N: N\left(x_{n}-x_{\ell}, z\right)_{p} \geq \varepsilon\right\} \in I$, for each $\varepsilon>0$ and non-zero $z \in X$. Let $P_{i}=\left\{n \in N: N\left(x_{n}-x_{m_{i}}, z\right)_{p}<\frac{1}{i}\right\}, i=1,2,3, \ldots$, where $m_{i}=N\left(\frac{1}{i}\right)$. Since $H_{i}=N-P_{i}=\left\{n \in N: N\left(x_{n}-x_{m_{i}}, z\right)_{p} \geq \frac{1}{i}\right\} \in I$ for each $i \in N$ and and non-zero $z \in X$, therefore $P_{i} \in F(I), i \in N$. Since $I$ has (AP) property then by Lemma (15) there exists a set $P \subset N$ such that $P \in F(I)$ and the set $P-P_{i}$ is finite for all $i$. Now we have to show that $\lim _{n, m \rightarrow \infty} N\left(x_{n}-x_{m}, z\right)_{p}=0, m, n \in P$ and for each non-zero $z$ in $X$. For this, let $\varepsilon>0$ and $j \in N$ be such that $j>\frac{2}{\varepsilon}$. If $m, n \in P$ then $P-P_{j}$ is a finite set, so there exists $k=k(j)$ such that $m \in P_{j}$ and $n \in P_{j}$ for all $m, n>k(j)$. Therefore $N\left(x_{n}-x_{m_{j}}, z\right)_{p}<\frac{1}{j}$ and $N\left(x_{m}-x_{m_{j}}, z\right)_{p}<\frac{1}{j}$, for all $m, n>k(j)$ and non-zero $z \in X$. Now $N\left(x_{n}-x_{m}, z\right)_{p}=N\left(x_{n}-x_{m_{j}}+x_{m_{j}}-x_{m}, z\right)_{p}=N\left(\left(x_{n}-x_{m_{j}}\right)-\right.$ $\left.\left(x_{m}-x_{m_{j}}\right), z\right)_{p} \leq N\left(x_{n}-x_{m_{j}}, z\right)_{p}+N\left(x_{m}-x_{m_{j}}, z\right)_{p}<\frac{1}{j}+\frac{1}{j}=\frac{2}{j}<2 \frac{\varepsilon}{2}=\varepsilon$, for $m, n>k(j)$ and each non-zero $z \in X$. Hence, for any $\varepsilon>0$ there exists $k=k(\varepsilon)$ such that for $m, n>k(\varepsilon)$ and $m, n \in P \in F(I), N\left(x_{n}-x_{m}, z\right)_{p}<\varepsilon$ for each non-zero $z \in X$. This shows that the sequence $\left\{x_{n}\right\}$ is $I^{*}$-Cauchy sequence 
in $X$. Thus $\left\{x_{n}\right\}$ is $I$-Cauchy sequence in $X$ if and only if $\left\{x_{n}\right\}$ is $I^{*}$-Cauchy sequence in $X$.

\section{References}

[1] G. Bachman, Introduction to p-Adic Numbers and Valuation Theory, Academic Press, (1964).

[2] G. Bachman and L. Narici, Functional Analysis, Academic Press, New York and London, (1966).

[3] A.J. Baker, An Introduction to p-Adic Numbers and p-Adic Analysis, URL: http:// www.maths.gla.ac.uk/_ajb.

[4] B. Dragovich, p-adic approach to the genetic code and genome, Institute of Physics, Belgrade, Serbia, TAG, 20-24 Oct, (2008), Annecy.

[5] S. Gähler, Linear 2-normerte raume, Math. Nachr, 28 (1965), 1-45.

[6] M. Gurdal and S. Pehlivan, The statistical convergence in 2-banach spaces, Thai J. Math., 2(1) (2004), 107-113.

[7] M. Gurdal, On ideal convergent sequences in 2-normed spaces, Thai Journal of Mathematics, 4(1) (2006), 85-91.

[8] M. Gurdal and Isil Acik, On I-Cauchy sequences in 2-normed spaces, Mathematical Inequalities and Applications, 11(2), (2008), 349-354

[9] H. Gunawan and Mashadi, On finite dimensional 2-normed spaces, Soochow J. Math., 27(3) (2001), 321-329.

[10] K. Hensel, Theorie der Algebraischen Zahlen, Teubner, Leipzig, (1908).

[11] S. Katok, Real and p-adic analysis course notes for math 497C mass program, Fall 2000 Revised, The Pennsylvania State University, University Park, PA 16802, U.S.A., November (2001).

[12] N. Koblitz, p-Adic Numbers, p-Adic Analysis and Zeta-Functions (2nd ed.), New York: Springer-Verlag, (1984).

[13] C.K. Koc, A tutorial on p-adic arithmetic, electrical and computer engineering, Oregon State University Corvallis, Oregon 97331, Technical Report, April (2002). 
[14] P. Kostyrko, M. Macaj and T. Salat, I-convergence, Real Anal. Exchange, 26(2) (2000), 669-686.

[15] P. Kostyrko, M. Macaj, T. Salat and M. Sleziak, I-convergence and extremal I-limit points, Math. Slovaca, 55 (2005), 443-464.

[16] M. Kurt, Introduction to p-Adic Numbers and their Functions (2nd ed.), Cambridge, England: Cambridge University Press, (1981).

[17] Z. Lewandowska, Generalized 2-normed spaces, Stuspskie Prace Matematyczno-Fizyczne, 1 (2001), 33-40.

[18] Z. Lewandowska, Linear operators on generalized 2-normed spaces, Bull. Math. Soc. Sci. Math, Roumanie (N.S.), 42(90) (4) (1999), 353-368.

[19] Z. Lewandowska, On 2-normed sets, Glasnik Mat. Ser. III, 38(58) (1) (2003), 99-110.

[20] Mehmet Acikgoz, N. Aslan, N. Koskeroglu and S. Araci, p-adic approach to linear 2-normed spaces, Mathematica Moravica, 13(2) (2009), 7-22.

[21] Anar Nabiev, Serpil Pehlivan and M. Gurdal, On I-Cauchy sequences , Taiwanese Journal of Mathematics, 11(2), June 2007, 569-576.

[22] W. Raymond, R. Freese and J. Cho, Geometry of Linear 2-Normed Spaces, Nova Science Publishers, (2001).

[23] A.M. Robert, A course in p-adic analysis, Graduate Texts in Mathematics, 198 (2000), 1-440.

[24] H. Sahiner, M. Gurdal, S. Saltan and H. Gunawan, Ideal convergence in 2-normed spaces, Taiwanese Journal of Mathematics, 11(5) (2007), 14771484 .

[25] B.Surender Reddy, Equivalence of p-adic 2-norms in p-adic linear 2-normed spaces, International Journal of Open Problems in Computer Science and Mathematics, 3(5) (2010), 25-38.

[26] B.Surender Reddy and D.Shankaraiah, On Ideal convergent sequences in p-adic linear 2-normed spaces, General Mathematics Notes, 17(1), July 2013, 88-104.

[27] V.S. Vladimirov, I.V. Volovich and E.I. Zelenov, p-Adic Analysis and Mathematical Physics, World Scientific, Singapore, (1994). 
[28] S.S. William, Notes on p-Adic Numbers, Rice University, Houston, Texas, (2005). 